Pacific Journal of Mathematics

ON BOUNDARY FUNCTIONALS AND OPERATORS WITH 


\title{
ON BOUNDARY FUNCTIONALS AND OPERATORS WITH FINITE-DIMENSIONAL NULL SPACES
}

\author{
FRANKLIN T. IHA
}

Let $L$ be a closed operator on a Hilbert space $\mathscr{H}$ defined on a linear manifold $\mathscr{D}$ of $\mathscr{H}$ with the property that $L$ has a continuous right inverse $T$ and that the dimension of the null space of $L$ is finite. A boundary functional $\eta$ for $L$ is defined to be a linear functional $\eta$ on $\mathscr{D}$ such that $\eta T$ is continuous. The boundary-value problems for ordinary differential equations are generalized to the operator $L$ with the boundary conditions defined by a set of boundary functionals. It is shown, in particular, that if $K$ is a continuous right inverse of $L$, then there exist $n$ linearly independent boundary functionals, $\eta_{1}, \cdots, \eta_{n}$, where $n$ is the dimension of the null space of $L$, such that the range of $K$ is precisely the linear manifold $\left\{u \in \mathscr{D} \mid \eta_{j}(u)=0, j=1,2, \cdots, n\right\}$.

We make a further abstraction on the generalized homogeneous boundary-value problems of ordinary differential equations treated in [4]. Moreover, we prove that the condition imposed in [4] on the linear functionals defining the boundary conditions is not only sufficient but necessary in order to obtain a continuous right inverse.

In earlier works concerning generalized boundary-value problems, such as found in the survey article [5] by Whyburn, no consideration is given to the continuity of the right inverse of the differential operator. Yet, if we examine how boundary-value problems arise in application, we see that the right-hand side of the the equation $L y=f$ is usually a force function which is at best an approximate description of the force acting on the physical system. Thus, without the consideration of the continuity of the right inverse, the solution of a boundaryvalue problem must be regarded as incomplete. It is interesting to note that in all the boundary-value problems arising in application involving an $n$th order differential equation, conditions are never imposed on the $n$th derivative. From equation (8) of [4], we see that the linear functional $\eta$ defined by $\eta(u)=u^{(n)}(c), u \in C^{n}([a, b])$, where $c \in[a, b]$, does not have the property that $\eta T$ is continuous (with respect to the $L^{2}$ norm), where $T$ is a continuous right inverse of the differential operator $L$. We show in this paper that the linear functional $\eta$ must have the property that $\eta T$ is continuous in order to obtain a continuous right inverse. Thus, even if such a boundary condition did arise in application, the solution of the boundary-value problem would not have been usable. 
Boundary-value problems considered here are actually much more general than the usual generalized boundary-value problems for a single $n$th order equation. Very little use is made of the properties of differential operators, and consequently, the treatment is set in the language of a Hilbert space. Similar abstraction of the generalized boundary-value problems for systems of differential equations considered by such authors as Whyburn [5], Cole [3], and Bryan [2] can possibly be made, but it is not undertaken here.

2. Notations, definitions and a lemma. Throughout this paper $\mathscr{H}$ denotes a Hilbert space and $L$ a closed operator from $\mathscr{D} \subset \mathscr{H}$ into $\mathscr{H}$, where $\mathscr{D}$ is a linear manifold not necessarily dense in $\mathscr{H}$. We let

$$
\mathscr{S}=\{u \in \mathscr{D} \mid L u=0\}
$$

and we assume $\operatorname{dim} \mathscr{S}=n<\infty$. We also assume that there exists a linear operator $T$ on $\mathscr{H}$ having the following properties:

$$
\left\{\begin{array}{c}
L T f=f \text { for all } f \in \mathscr{H} \\
\|T f\| \leqq c\|f\| \text { for all } f \in \mathscr{H},
\end{array}\right.
$$

where $c$ is a constant. Following the terminology used in [4], we make the following definitions.

Definition 1. A linear functional $\eta$ defined on $\mathscr{D}$ is said to be a boundary functional for $L$ if $\eta T$ is continuous.

Definition 2. A set $\left\{\eta_{1}, \cdots, \eta_{l}\right\}$ of boundary functionals is said to be linearly independent if the set of their restrictions to $\mathscr{S}$ is linearly independent, that is, if $\sum_{l=1}^{l} \alpha_{j} \eta_{j}(u)=0$ for all $u \in \mathscr{S}$ implies $\alpha_{\jmath}=0$ for all $j, 1 \leqq j \leqq l$; or equivalently, if for any basis $\left\{u_{1}, \cdots, u_{n}\right\}$ of $\mathscr{S}$ the rank of the matrix $\left[\eta_{i}\left(u_{j}\right)\right]$ is $l$.

The following lemma shows that the property of a linear functional $\eta$ being a boundary functional for $L$ depends only on $L$. Because of the rather remarkable property, we give the lemma in a slightly more general form than needed in this paper.

Lemma. Let $L, \mathscr{D}$, and $\mathscr{S}$ be as defined above. Let $L$ have an additional property that $\operatorname{dim} \mathcal{N}(L-\lambda I)<\infty$ for all $\lambda \in \mathbf{C}$, where $\mathcal{N}(L-\lambda I)$ is the null space of $L-\lambda I$ and $\mathbf{C}$ is the set of all complex numbers. Let $\lambda_{1}, \lambda_{2} \in \mathbf{C}$ and $K_{1}$ and $K_{2}$ be continuous right inverses of $L-\lambda_{1} I$ and $L-\lambda_{2} I$, respectively. If $\eta$ is a linear functional defined on $\mathscr{D}$ such that $\eta K_{1}$ is continuous, then $\eta K_{2}$ is also continuous. 
Proof. Let $u \in \mathscr{D}$. Then,

$$
\left(L-\lambda_{1} I\right)\left[K_{1}\left(L-\lambda_{1} I\right) u-u\right]=0
$$

so that

$$
K_{1}\left(L-\lambda_{1} I\right) u-u \in \mathcal{N}\left(L-\lambda_{1} I\right) .
$$

Since $K_{2} \mathscr{H} \subset \mathscr{D}$, it follows

$$
K_{1}\left(L-\lambda_{1} I\right) K_{2} f-K_{2} f \in \mathcal{N}\left(L-\lambda_{1} I\right)
$$

for all $f \in \mathscr{H}$.

Let $\left\{z_{1}, \cdots, z_{m}\right\}$ be an orthonormal basis for $\mathcal{N}\left(L-\lambda_{1} I\right)$. Then,

$$
K_{1}\left(L-\lambda_{1} I\right) K_{2} f-K_{2} f=\sum_{j=1}^{m} \alpha_{j} z_{j}
$$

where

$$
\alpha_{j}=\left(K_{1}\left(L-\lambda_{1} I\right) K_{2} f-K_{2} f, z_{j}\right), \quad j=1, \cdots, m .
$$

Since $\left(L-\lambda_{1} I\right) K_{2} f=f+\left(\lambda_{2}-\lambda_{1}\right) K_{2} f$, we see that the map $\mathscr{E}_{k}: f \rightarrow \alpha_{k}$ is continuous. From (3) we obtain

$$
\eta\left(K_{2} f\right)=\eta\left(K_{1}\left(L-\lambda_{1} I\right) K_{2} f\right)-\sum_{j=1}^{m} \mathscr{E}_{j}(f) \eta\left(z_{j}\right)
$$

From this and from the continuities of $K_{1}, K_{2}, \mathscr{E}_{k}$ and $\left(L-\lambda_{1} I\right) K_{2}$, the continuity of $\eta K_{2}$ follows.

We use this lemma essentially for the case $\lambda_{1}=\lambda_{2}=0$, and for this case we do not need the assumption that $\operatorname{dim} \mathcal{N}\left(L-\lambda_{1} I\right)<\infty$ for all $\lambda \in \mathbf{C}$.

\section{Generalized boundary-value problems and spec-} tral theorem. We begin with a theorem which is an immediate generalization of Theorem 1 of [4] and can be proved in the same way.

Theorem 1. Let $L, \mathscr{D}, \mathscr{S}$ and $T$ be as defined in $\$ 2$. Let $\left\{\eta_{1}, \cdots, \eta_{n}\right\}$ be a linearly independent set of boundary functionals for $L$, and let

$$
M=\left\{u \in \mathscr{D} \mid \eta_{j}(u)=0, \quad 1 \leqq j \leqq n\right\}
$$


Then there exists a continuous operator $K$ form $\mathscr{H}$ onto $\mathcal{M}$ such that $L K f=f$ for all $f \in \mathscr{H}$.

We now prove converses of this theorem.

THEOREM 2. Let $L, \mathscr{D}$ and $\mathscr{S}$ be as defined in $\S 2$. Let $\left\{\eta_{1}, \cdots, \eta_{n}\right\}$ be a set of linear functionals on $\mathscr{D}$ such that their restrictions to $\mathscr{S}$ are linearly independent. Let $\mathcal{M}=\left\{u \in \mathscr{D} \mid \eta_{\jmath}(u)=0,1 \leqq j \leqq n\right\}$. If the right inverse $K$, which does exist, from $\mathscr{H}$ into $M$ is continuous, then each $\eta$, must be a boundary functional for $L$.

Proof. By the definition of $K, K f \in \mathcal{M}$ for all $f \in \mathscr{H}$, and so $\eta_{l}(K f)=0$ for all $f \in \mathscr{H}$. Hence each $\eta_{l} K$ is trivially continuous and the assertion follows from the Lemma.

THEOREM 3. Let $L, \mathscr{D}$ and $\mathscr{S}$ be as defined in $\S 2$. If $K$ is a continuous operator from $\mathscr{H}$ into $\mathscr{D}$ such that $L K f=f$ for all $f \in \mathscr{H}$, then there exist $n$ linearly independent boundary functionals $\eta_{j}, j=1,2, \cdots, n$, such that

$$
\mathscr{R}(K)=\left\{u \mid \eta_{j}(u)=0, \quad 1 \leqq j \leqq n\right\}
$$

where $\mathscr{R}(K)$ is the range of $K$ and $n=\operatorname{dim} \mathscr{S}$.

Proof. Let $\left\{y_{1}, \cdots, y_{n}\right\}$ be an orthonormal basis for $\mathscr{S}$. Define $\eta_{j}$ by

$$
\eta_{j}(u)=\left(u-K L u, y_{j}\right), \quad u \in \mathscr{D}, \quad j=1,2, \cdots, n .
$$

Then, $\eta_{J}(K f)=0$ for all $f \in \mathscr{H}$, and so each $\eta_{j} K$ is trivially continuous. Thus, from the Lemma, each $\eta_{\text {, }}$ is a boundary functional for $L$.

Let $\alpha_{\jmath} \in \mathbf{C}$ such that $\sum_{j=1}^{n} \alpha_{\jmath} \eta_{\jmath}(u)=0$ for all $u \in \mathscr{S}$. Then, putting $\boldsymbol{y}_{k}$ for $u$, we obtain $\alpha_{k}=0, k=1, \cdots, n$. Hence, $\left\{\eta_{1}, \cdots, \eta_{n}\right\}$ is linearly independent in the sense of Definition 2. Let $\mathcal{M}=\left\{u \in \mathscr{D} \mid \eta_{j}(u)=0\right.$, $1 \leqq j \leqq n\}$. Let $u \in \mathscr{R}(K)$. Then $u=K f$ for some $f \in \mathscr{H}$ and so $\eta_{j}(u)=\eta_{j}(K f)=0$, for all $j, j=1, \cdots, n$. Hence, $u \in \mathcal{M}$ and $\mathscr{R}(K) \subset \mathcal{M}$.

Let $u \in \mathcal{M}$. Then

$$
\left(u-K L u, y_{j}\right)=\eta_{\jmath}(u)=0, \quad 1 \leqq j \leqq n .
$$

Hence, $u-K L u \in \mathscr{S}^{\perp}$, the orthogonal complement of $\mathscr{S}$. But $L(u-$ $K L u)=L u-L u=0$, so that $u-K L u \in \mathscr{P}$. Hence $u-K L u=0$ or $u=K(L u)$, so that $u \in \mathscr{R}(K)$ and so $\mathcal{M} \subset \mathscr{R}(K)$. Hence $\mathcal{M}=\mathscr{R}(K)$, which completes the proof. 
The question arises as to what happens if the set $\mathcal{M}$ is defined by two sets of linear functionals, one of which is a set of boundary functionals. The next result settles this question.

THEOREM 4. Let L, D and $\mathscr{S}$ be as defined in $\$ 2$ and assume $L$ has a continuous right inverse. Let $\left\{\eta_{1}, \cdots, \eta_{n}\right\}$ be a set of linearly independent boundary functionals for $L$ and let

$$
\mathcal{M}=\left\{u \in \mathscr{D} \mid \eta_{j}(u)=0, \quad 1 \leqq j \leqq n\right\} .
$$

If $\left\{\xi_{1}, \cdots, \xi_{n}\right\}$ is a set of linear functionals on $\mathscr{D}$ such that their restrictions to $\mathscr{S}$ are linearly independent and $M=\left\{u \in \mathscr{D} \mid \xi_{j}(u)=0\right.$, $1 \leqq j \leqq n\}$, then each $\xi_{i}$ must be a linear combination of $\eta_{j}$ 's.

Proof. Let $\left\{y_{1}, \cdots, y_{n}\right\}$ be an orthonormal basis for $\mathscr{S}$. Then the matrices $\left[\eta_{i}\left(y_{j}\right)\right]$ and $\left[\xi_{i}\left(y_{j}\right)\right]$ are invertible. So let $\left[\alpha_{i j}\right]$ and $\left[\beta_{i j}\right]$ be their inverses, respectively. Let

$$
\begin{gathered}
\zeta_{i}=\sum_{j=1}^{n} \alpha_{i j} \eta_{j} \\
\zeta_{i}^{\prime}=\sum_{j=1}^{n} \beta_{i j} \xi_{j} .
\end{gathered}
$$

Then, we have $\zeta_{i}\left(y_{j}\right)=\delta_{i j}$ and $\zeta_{i}^{\prime}\left(y_{j}\right)=\delta_{i j}, 1 \leqq i \leqq m, 1 \leqq j \leqq n$. Since $\left\{\eta_{1}, \cdots, \eta_{n}\right\}$ is a linearly independent set of boundary functionals for $L$, by Theorem 1 there exists a continuous right inverse $K$ of $L$ from $\mathscr{H}$ onto $\mathcal{M}$. Then $\eta_{j}(K f)=0=\xi_{\jmath}(K f)$ for all $f \in \mathscr{H}, j=1, \cdots, n$. Hence, $\zeta_{i}(K f)=\zeta_{i}^{\prime}(K f)=0$ for all $f \in \mathscr{H}, 1 \leqq i \leqq n$. Let $u \in \mathscr{D}$. Then $L u \in \mathscr{H}$, so that $K L u \in M$. Moreover, $u-K L u \in \mathscr{Y}$, and so $u=$ $K L u+\sum_{j=1}^{n} r_{l} y_{j}$ for some $r_{j} \in \mathbf{C}$. Hence it follows that

$$
\begin{aligned}
& \zeta_{i}(u)=r_{i} \quad \text { and } \\
& \zeta_{i}^{\prime}(u)=r_{i} .
\end{aligned}
$$

Thus, $\zeta_{l}(u)=\zeta_{i}^{\prime}(u)$ for all $u \in \mathscr{D}$, or $\sum_{j=1}^{n} \beta_{i j} \xi_{j}(u)=\sum_{j=1}^{n} \alpha_{i j} \eta_{j}(u)$ for all $u \in \mathscr{D}, i=1, \cdots, n$. Since the matrix $\left[\beta_{i j}\right]$ is invertible, it follows there exist $r_{i j} \in \mathbf{C}$ such that

$$
\xi_{i}(u)=\sum_{j=1}^{n} r_{i j} \eta_{j}(u), \quad u \in \mathscr{D}, \quad i=1,2, \cdots, m
$$

We want to state the immediate corollary of these theorems with respect to differential operators. Let $P(x, D)=\sum_{k=0}^{n} a_{k}(x) D^{k}$ and let $I$ 
be an interval, may be finite or infinite. If $a_{k}$ 's are sufficiently differentiable, i.e. $a_{k} \in C^{k}(I)$, then it can readily be shown that the operator $u \rightarrow P(x, D) u$ from $C_{0}^{\infty}(I) \subset L^{2}(I)$ into $L^{2}(I)$ is closable, where $C_{0}^{\infty}(I)$ is the set of all infinitely differentiable complex valued functions with compact support in $I$. Let $L$ be the closure of the operator $u \rightarrow P(x, D) u, u \in C_{0}^{\infty}(I)$ in $L^{2}(I)$. Let $\mathscr{D}$ be the domain of definition of $L, \mathscr{S}$ the null space of $L$ and $\operatorname{dim} \mathscr{S}=n$. With these definitions, we have

COROllary. If $L$ has a continuous right inverse $T$ and if $\left\{\eta_{1}, \cdots, \eta_{n}\right\}$ is a linearly independent set of boundary functionals, then there exists a continuous right inverse $K$ of $L$ such that

$$
\mathscr{R}(K)=\left\{u \in \mathscr{D} \mid \eta_{,}(u)=0, \quad 1 \leqq j \leqq n\right\} .
$$

Conversely, if $K$ is a continuous right inverse of $L$, then there exists a linearly independent set of boundary functionals, $\eta_{1}, \cdots, \eta_{n}$, such that

$$
\mathscr{R}(K)=\left\{u \in \mathscr{D} \mid \eta_{j}(u)=0, \quad 1 \leqq j \leqq n\right\} .
$$

Moreover, if $\left\{\xi_{1}, \cdots, \xi_{n}\right\}$ is a set of linear functionals on $\mathscr{D}$ whose restrictions to $\mathscr{S}$ are linearly independent and

$$
\mathscr{R}(K)=\left\{u \in \mathscr{D} \mid \xi_{j}(u)=0, \quad 1 \leqq j \leqq n\right\},
$$

then each $\xi_{2}$ must be a linear combination of $\eta_{j}$ 's.

We now put Theorem 5 of [4] in the general setting, keeping in mind a possible application to singular boundary-value problems which were not treated in [4]. Since a part of the proof is almost exactly the same as that of Theorem 5 of [4], that part of the proof will only be sketched.

TheOREM 5. Let L, H, $\mathscr{D}$ and $\mathscr{S}$ be as defined in $\$ 2$, and assume that $L$ has a continuous right inverse. Let $\left\{\eta_{1}, \cdots, \eta_{l}\right\}$ be a set of boundary functionals in which $\left\{\eta_{1}, \cdots, \eta_{r}\right\}$ is linearly independent in the sense of Definition 2, and let

$$
\mathcal{M}_{0}=\left\{u \in \mathscr{D} \mid \eta_{j}(u)=0, \quad 1 \leqq j \leqq l\right\}
$$

Let $\mathcal{N}=\mathscr{S} \cap \mathcal{M}_{0}$, so that $\operatorname{dim} \mathcal{N}=p=n-r$ and let $q=l-r . \quad$ Let $\left\{\psi_{1}, \cdots, \psi_{p}\right\}$ be an orthonormal basis for $\mathcal{N}$. Define $\zeta_{j}$ by

$$
\zeta_{j}(f)=\left(f, \psi_{i}\right), \quad f \in \mathscr{H}, \quad j=1, \cdots, p,
$$




$$
M_{1}=\left\{u \in \mathscr{D} \mid \eta_{\jmath}(u)=\zeta_{k}(u)=0, \quad 1 \leqq j \leqq r, \quad 1 \leqq k \leqq p\right\}
$$

Then there exists a continuous right inverse $K$ of $L$ from $\mathscr{H}$ onto $\mathcal{M}_{1}$. If moreover, $L$ is self-adjoint on $\mathcal{M}_{0}$, that is, if $(L u, v)=(u, L v)$ for all $u, v \in \mathcal{M}_{0}$, then there exists a spectral measure $E$ such that

$$
K f=\left(\int \lambda d E\right) f+\sum_{j=1}^{q}\left(f, \psi_{j}\right) K f, \quad f \in \mathscr{H} .
$$

Proof. It can readily be shown that $\left\{\eta_{1}, \cdots, \eta_{r}, \zeta_{1}, \cdots, \zeta_{p}\right\}$ is a linearly independent set of boundary functionals. Hence the existence of the continuous right inverse $K$ of $L$ from $\mathscr{H}$ onto $\mathcal{M}_{1}$ follows from Theorem 1. To prove the second part, we first show, just as in the proof of Theorem 5 of [4], that every $f \in \mathscr{H}$ can be written as

$$
f=g+\sum_{j=1}^{q}\left(f, \psi_{j}\right) \psi_{j}
$$

with $g \in L\left(\mathcal{M}_{0} \cap \mathcal{M}_{1}\right)$, the image of $\mathcal{M}_{0} \cap \mathcal{M}_{1}$ under $L$. From this we can show that $L\left(\mathcal{M}_{0} \cap \mathcal{M}_{1}\right)$ is invariant under $K$.

It can readily be shown that $L\left(\mathcal{M}_{0} \cap \mathcal{M}_{1}\right)$ is a closed subspace of $\mathscr{H}$. Hence if we denote by $K_{0}$ the restriction of $K$ to $L\left(\mathcal{M}_{0} \cap \mathcal{M}_{1}\right), K_{0}$ is bounded self-adjoint operator on the Hilbert space $L\left(\mathcal{M}_{0} \cap \mathcal{M}_{1}\right)$ and so there exists a spectral measure $E_{0}$, whose values are projection operators on $L\left(\mathcal{M}_{0} \cap \mathcal{M}_{1}\right)$, such that

$$
K_{0} f=\left(\int \lambda d E_{0}\right) f, \quad f \in L\left(M_{0} \cap M_{1}\right) .
$$

Let $P$ be the orthogonal projection of $\mathscr{H}$ onto $L\left(\mathcal{M}_{0} \cap \mathcal{M}_{1}\right)$. If $\delta$ is a Borel set in $\sigma\left(K_{0}\right)$, the spectrum of $K_{0}$, define $E(\delta)=E_{0}(\delta) P$. Then $E$ becomes a spectral measure in the usual sense (c.f. [1]). Let $f \in \mathscr{H}$ and let

$$
g=f-\sum_{j=1}^{q}\left(f, \psi_{1}\right) \psi_{j}
$$

Then, it follows from (5) that

$$
K g=\left(\int \lambda d E_{0}\right) g=\left(\int \lambda d E_{0}\right) P f=\left(\int \lambda d E\right) f
$$

so that

$$
K f=\left(\int \lambda d E\right) f+\sum_{j=1}^{q}\left(f, \psi_{j}\right) K \psi_{j}
$$




\section{REFERENCES}

1. S. K. Berberian, Notes on Spectral Theory, Van Nostrand Mathematical Studies, D. Van Nostrand Company, Inc. New York, 1966.

2. R. N. Bryan, A linear differential system with generalized linear boundary conditions, J. Differential Equations, 5 (1969), 38-48.

3. R. H. Cole, General boundary conditions for an ordinary differential system, Trans. Amer. Math. Soc., 111 (1964), 521-550.

4. F. T. Iha, A unified approach to boundary value problems on compact intervals, Pacific J. Math., to appear.

5. W. M. Whyburn, Differential equations with general boundary conditions, Bull. Amer. Math. Soc., 48 (1942), 692-704.

Received November 28, 1973.

UNIVERSITY OF HAWAII 


\section{PACIFIC JOURNAL OF MATHEMATICS \\ EDITORS}

\author{
Richard ARENS (Managing Editor) \\ University of California \\ Los Angeles, California 90024
}

\author{
R. A. Beaumont \\ University of Washington \\ Seattle, Washington 98105
}

\section{J. DugundJI}

Department of Mathematics

University of Southern California

Los Angeles, California 90007

D. Gilbarg and J. Milgram

Stanford University

Stanford, California 94305

\section{ASSOCIATE EDITORS}
E. F. BECKENBACH
B. H. NeumanN
F. WOLF
K. YoshidA

\section{SUPPORTING INSTITUTIONS}

\author{
UNIVERSITY OF BRITISH COLUMBIA \\ CALIFORNIA INSTITUTE OF TECHNOLOGY \\ UNIVERSITY OF CALIFORNIA \\ MONTANA STATE UNIVERSITY \\ UNIVERSITY OF NEVADA \\ NEW MEXICO STATE UNIVERSITY \\ OREGON STATE UNIVERSITY \\ UNIVERSITY OF OREGON \\ OSAKA UNIVERSITY
}

\author{
UNIVERSITY OF SOUTHERN CALIFORNIA \\ STANFORD UNIVERSITY \\ UNIVERSITY OF TOKYO \\ UNIVERSITY OF UTAH \\ WASHINGTON STATE UNIVERSITY \\ UNIVERSITY OF WASHINGTON \\ AMERICAN MATHEMATICAL SOCIETY
}

The Supporting Institutions listed above contribute to the cost of publication of this Journal, but they are not owners or publishers and have no responsibility for its contents or policies.

Mathematical papers intended for publication in the Pacific Journal of Mathematics should be in typed form or offset-reproduced (not dittoed), double spaced with large margins. Underline Greek letters in red, German in green, and script in blue. The first paragraph or two must be capable of being used separately as a synopsis of the entire paper. Items of the bibliography should not be cited there unless absolutely necessary, in which case they must be identified by author and Journal, rather than by item number. Manuscripts, in duplicate, may be sent to any one of the four editors. Please classify according to the scheme of Math. Reviews, Index to Vol. 39. All other communications should be addressed to the managing editor, or Elaine Barth, University of California, Los Angeles, California, 90024.

100 reprints are provided free for each article, only if page charges have been substantially paid. Additional copies may be obtained at cost in multiples of 50 .

The Pacific Journal of Mathematics is issued monthly as of January 1966. Regular subscription rate: $\$ 72.00$ a year (6 Vols., 12 issues). Special rate: $\$ 36.00$ a year to individual members of supporting institutions.

Subscriptions, orders for back numbers, and changes of address should be sent to Pacific Journal of Mathematics, 103 Highland Boulevard, Berkeley, California, 94708.

PUBLISHED BY PACIFIC JOURNAL OF MATHEMATICS, A NON-PROFIT CORPORATION Printed at Jerusalem Academic Press, POB 2390, Jerusalem, Israel.

$$
\begin{gathered}
\text { Copyright (c) } 1975 \text { Pacific Journal of Mathematics } \\
\text { All Rights Reserved }
\end{gathered}
$$




\section{Pacific Journal of Mathematics}

Vol. 56, No. 2 December, 1975

Ralph Alexander, Generalized sums of distances .................. 297

Zvi Arad and George Isaac Glauberman, A characteristic subgroup of a group of odd order ............................... 305

B. Aupetit, Continuité du spectre dans les algèbres de Banach avec involution ........................................ 321

Roger W. Barnard and John Lawson Lewis, Coefficient bounds for some classes of starlike functions . ...........................

Roger W. Barnard and John Lawson Lewis, Subordination theorems for some classes of starlike fumctions . .................... 333

Ladislav Bican, Preradicals and injectivity .................. 367

James Donnell Buckholtz and Ken Shaw, Series expansions of analytic

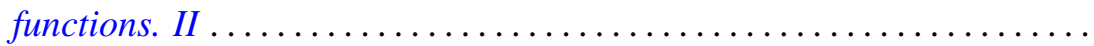

Richard D. Carmichael and E. O. Milton, Distributional boundary values in

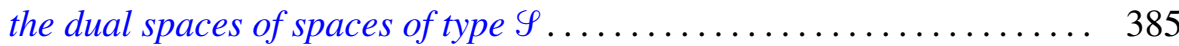

Edwin Duda, Weak-unicoherence ............................ 423

Albert Edrei, The Padé table of functions having a finite number of essential singularities ........................................

Joel N. Franklin and Solomon Wolf Golomb, A function-theoretic approach to the study of nonlinear recurring sequences ................ 455

George Isaac Glauberman, On Burnside's other $p^{a} q^{b}$ theorem 469

Arthur D. Grainger, Invariant subspaces of compact operators on topological vector spaces .........................

Jon Craig Helton, Mutual existence of sum and product integrals .

Franklin Takashi Iha, On boundary functionals and operators with finite-dimensional null spaces

Gerald J. Janusz, Generators for the Schur group of local and global number fields

A. Katsaras and Dar-Biau Liu, Integral representations of weakly compact operators.

W. J. Kim, On the first and the second conjugate points .

Charles Philip Lanski, Regularity and quotients in rings with involution ....

Ewing L. Lusk, An obstruction to extending isotopies of piecewise linear manifolds.

Saburou Saitoh, On some completenesses of the Bergman kernel and the Rudin kernel..

Stephen Jeffrey Willson, The converse to the Smith theorem for $Z_{p}$-homology spheres. 